\title{
Adsorptive SPH for Directable Bleeding Simulation
}

\author{
Kazuhide Ueda* ${ }^{*}$ Issei Fujishiro ${ }^{\dagger}$ \\ Keio University
}

\begin{abstract}
We build upon SPH to devise a directable scheme for bleeding simulation that realistically represents adherence onto and streak formation on the skin surface in real time. To ensure compatibility of expressiveness and space \& time efficiency, we have judiciously designed four specific types of particles and their interactions and incorporated these elements into the SPH scheme. In-vivo particles obey a simple in-vivo blood flow model that can reflect both hemorrhage volume and blood pressure. The particles flowing out through the wound, called flow particles, manifest the primary behaviors of bleeding, including blood coagulation, blood adherence onto the skin surface, and streak formation on the skin surface. The scheme then involves transforming the flow particles adhered onto the skin surface into relatively small adhered particles and representing a thin, flat layer of adhered blood with anisotropic kernels. Guiding particles generated along user-input strokes play a distinct role in giving gravity, biased slightly by the guiding force, to the remaining flow particles and thereby allow the users to control the overall bleeding so that it follows the desirable traces.
\end{abstract}

CR Categories: I.3.7 [Computer Graphics]: Three-Dimensional Graphics and Realism-Animation; I.3.5 [Computer Graphics]: Computational Geometry and Object Modeling-Physically based modeling.

Keywords: Fluid simulation, SPH, adsorption, guide, bleeding.

\section{Introduction}

Fluid simulation has been extensively used to produce various types of special effects (SFX) in the film and computer game industries. Physically-plausible liquid behavior has a significant impact on virtual worlds, and bleeding is no exception. Blood possesses highly important implications, specially considering that films and games involving hemorrhage scenes might receive age-restricting ratings. Insufficient visual reality in these types of scenes would reduce the overall quality of the work.

Keeping this background in mind, we have built upon a common Lagrangian method called SPH (Smoothed Particle Hydrodynamics) to simulate bleeding faithfully. Our thorough feature analysis has clarified that the main features of bleeding cover a broad range of fields, from fluid physics - including streak formation due to adhesion and coagulation - to optics, which plays into light scattering and absorption inside participating media.

Blood consists mainly of solid components, such as red blood cells, white blood cells, platelets, and blood plasma. Platelets play a role in plugging up and repairing damaged blood vessels and wounds.

*e-mail: uk0408@gmail.com

†e-mail: fuji@ics.keio.ac.jp

Permission to make digital or hard copies of all or part of this work for personal or classroom use is granted without fee provided that copies are not made or distributed for profit or commercial advantage and that copies bear this notice and the full citation on the first page. Copyrights for components of this work owned by others than ACM must be honored. For all other uses, contact the Owner/Author.

Copyright is held by the owner/author(s).

VRCAI '15, October 30 - November 1, 2015, Kobe, Japan.

ACM 978-1-4503-3940-7/15/10.

http://dx.doi.org/10.1145/2817675.2817684
Coagulation is another effect of platelets, which react with fibrin and other clotting factors absorbed in blood plasma to generate blood clots and thereby stop bleeding. The adherence of liquid onto an object and sliding evenly underneath the lower-horizontal surface of the object can be explained as a sort of physisorption caused by van der Waals force. Flowing blood results in streaks on the object surface due to the viscous force of adhered blood.

Given these preliminary considerations, we judiciously designed specific types of bleeding particles and their interactions and incorporated them into the original SPH scheme. If we were to use a common definition of particle variables throughout the entire simulation, most of the particles would thus need to maintain unnecessary properties at most time-steps; in turn, this would waste the limited memory available.

Specifically, we employ in-vivo particles for a simple SPH-based simulation of in-vivo blood flow. The underlying model takes both hemorrhage volume and bleed momentum into account and makes it possible to represent a variety of bleeding, including non-uniform blood streaks and blood splash. We redefine the in-vivo particles flowing out through the user-specifiable wound as flow particles, which serve to manifest the primary behaviors of bleeding: the method makes it possible to represent the coagulation of blood, for example, by changing the viscous force of each flow particle according to air exposure time.

Flow particles also relate to the adherence of blood to the skin surface. Each flow particle adhered to the skin surface is transformed into a larger population of adhered particles with relatively small radii. A thin, flat layer of adhered blood, represented with anisotropic kernels, then maintains a proper influence on the dynamics of the upper layer of adhered particles.

Recent literature on visual simulation makes frequent mention of the idea of "artistic directability". We also define guiding particles, which have an effect on the SPH-based particles, for this purpose. However, if we were to add a force from the guiding particles directly to the SPH particles, it would be challenging to render the resulting liquid behaviors in a physically-accurate manner - especially if the guiding direction were to conflict significantly with the direction of gravity. To make the fluid control more natural, we tilt the gravity of each particle by the guiding force and thereby allow the user to control the overall bleeding along the desired traces or circumvent predefined significant marks.

Red blood cells account for 96 percent of all total blood cells. Blood looks red due to the blood pigments of hemoglobin, which is combinable with oxygen. As these red blood cells absorb and scatter incident light, blood manifests its visual features as participating media. We decided to render blood simply as depth-sensitive participating media via a dedicated shader program without sacrificing any of the efficiency of the above simulation scheme.

In order to control blood interactively from the artistic point of view, we also implemented most of the entire codes for simulation and rendering on the GPU. Our GPU-assisted parallelized simulation scheme minimizes the frequency of bottleneck data transfers from the GPU to the CPU and makes it possible to direct bleeding interactively with prompt feedbacks.

Although our current design policy is to trade physical accuracy for interactivity, more precise physiological considerations, such 
as hemorrhage volume and coagulation speed, would extend the present scheme to possible applications in medical education, virtual surgery, and forensic analysis. The present scheme also readily lends itself to real-time simulation of fluid with the same physical features as blood, such as paint, sweat, and squashed fruit.

The rest of this paper is organized as follows. After the next section surveys the related work, Section 3 gives an overview of our simulation framework. Section 4 focuses on more detailed descriptions of physisorption and guiding in the bleeding simulation, and Section 5 explains how to implement real-time simulation codes with the aid of the GPU. Section 6 provides experimental results and discussions on the expressiveness and efficiency of the simulation. Finally, we conclude the paper in Section 7.

\section{Related Work}

[Lucy 1977] and [Gingold and Monaghan 1977] originally developed the SPH method for the simulation of astrophysical problems. The SPH relies on particles to sample the target space and simulate associated complex natural phenomena.

[Reeves 1983] introduced particle systems to the field of computer graphics for the first time. [Miller and Pearce 1989] imported ideas from molecular dynamics to add basic interparticle interactions. [Desbrun and Gascuel 1996], meanwhile, applied the SPH concepts to computer graphics in order to simulate highly-deformable bodies. [Müller et al. 2003] implemented interactive SPH-based liquid simulation and rendering with the CPU, simulating surface tension with ideas from [Morris 2000]. [Solenthaler and Pajarola 2009] presented incompressible fluid simulation using a prediction-correction scheme to determine particle pressure values.

To accelerate the SPH computation process, [Amada et al. 2004] used the GPU for the purpose of parallelization. In this study, the researchers computed the neighbor search on the CPU and performed the standard SPH-based physics computation on the GPU. [Ihmsen et al. 2011] presented an efficient system for computing SPH fluid simulations on multi-core CPUs. An entirely GPU-based SPH computation was proposed in [Harada 2007; Zhang et al. 2008], where a grid-based structure for simplifying the neighbor search unfortunately uses too much memory for buckets. [Zhou et al. 2008] presented another method to enable the efficient construction of KD-trees on the GPU, but hierarchical data structures are not necessarily suitable for SPH acceleration. The sliced data structure proposed by [Harada et al. 2007] also might use as much memory as the grid volume itself in the worst case. To avoid such excessive use of GPU memory, [Goswami et al. 2010] presented spatial indexing and searching based on Z-indexing, which eliminates the use of buckets and makes it possible to determine the neighborhood set of particles on a constant basis. The researchers also rendered liquid at a high frame rate using GPU-based raycasting.

Bleeding is a typical example of fluid appressed to an object surface, whose forces have a great influence on the dynamics of the fluid. [Wang et al. 2005] modeled surface tensions between liquids and solid objects to solve the capillary solid coupling problem and animated small-scale fluid phenomena including water drops on surfaces. [Wang et al. 2007] simulated small-scale 3D water flows on solid surfaces under shallow wave assumptions. [Zhang et al. 2011], meanwhile, presented a deformable surface model that also provides a basis for animating the small-scale fluid motion of water drops. [Clavet et al. 2005] added an attraction force to make particles remain even underneath a horizontal surface. However, this force alone is not enough to represent surface adhesion properly. Therefore, we decided to gener- ate adhered particles when flow particles collide with the surface. [Schechter and Bridson 2012] proposed a ghost fluid approach to allow for free surface and solid boundary conditions.

[Ueda and Fujishiro 2012] simulated the adherence of blood to the skin surface. When detecting a particle penetrating into the skin surface, the particle was forced by the method to stay at the surface. However, the volume of the blood that SPH represents is greater than that of adhered blood in reality; the gross volume of blood thus dwindles to an extremely low level, preventing the blood from flowing smoothly.

When simulating adhered particles and flow particles simultaneously, one needs to take the differences in their sizes into account. [Adams et al. 2007] used a small number of particles to save on computational costs while maintaining the same level of quality by changing particle size adaptively. Some spatial difficulties in splitting and merging particles still persist, however. [Solenthaler and Gross 2011] presented a two-scale method to address this problem by dividing the simulation area into three parts.

To represent a thin, flat liquid surface, [Yu and Turk 2010] used anisotropic smoothing kernels. We also adopted a similar approach to represent a thin, flat layer of adhered blood.

If users applying fluid simulations in video production can control the fluid behavior freely, the quality of work improves dramatically. [Thürey et al. 2006] controlled the fluid while keeping its details by incorporating the small-scale velocity field, solvable with SPH, into grid-based fluid simulation. [Kim et al. 2006] guided smoke with grid-based fluid simulation by generating velocity fields based on user-input strokes.

Several studies have examined virtual surgery involving blood simulation [Xue-mei et al. 2009; Daenzer et al. 2006]. [Müller et al. 2004] simulated blood flow in vessels for a virtual surgery system. Although the study simulated bleeding from blood vessel walls interactively, the researchers did not consider the visual reality of blood. Blood demonstrates optical features as participating media due to light absorption and the scattering of light by red blood cells. [Frisvad et al. 2007] rendered realistic participating media through an application of the Lorenz-Mie theory at the cost of expensive computation for optically accurate subsurface scattering. [Yim et al. 2012] described a predictive model of light interaction with human blood that provided spectral and scattering responses for blood samples under a wide range of physiological and rheological conditions. We decided to render blood simply as depth-sensitive participating media with a dedicated shader program, making an effort not to undermine the efficiency of the simulation codes.

\section{Visual Simulation of Bleeding}

As we mentioned earlier, we rely on SPH as the underlying fluid simulation solver. Field quantity $A$ can be evaluated anywhere in space by summing up the contributions of neighboring particles:

$$
A(\mathbf{x})=\sum_{j} A_{j} \frac{m_{j}}{\rho_{j}} W\left(\mathbf{x}-\mathbf{x}_{j}\right),
$$

where $\mathbf{x}$ denotes the position, $m_{j}$ the mass of particle $j, \rho$ the density, and $W\left(\mathbf{x}-\mathbf{x}_{j}\right)$ the isotropic smoothing kernel.

The governing equations for fluids are the mass conservation equation and the momentum conservation equation (Navier-Stokes equations):

$$
\frac{D \rho}{D t}+\rho \cdot \mathbf{v}=0
$$




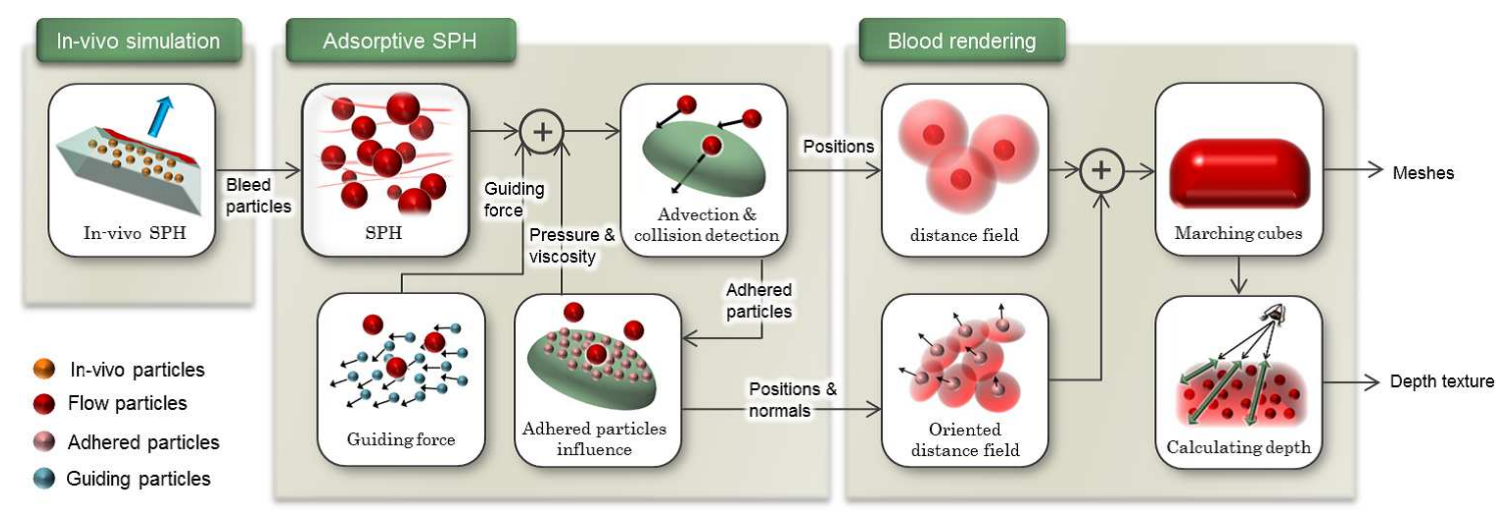

Figure 1: The dataflow of bleeding simulation on the GPU; each stage invokes various methods (the rounded squares). The number of threads allocated on the GPU differs according to the methods invoked.

$$
\rho \frac{D \mathbf{v}}{D t}=\mathbf{f}^{\text {pressure }}+\mathbf{f}^{\text {viscosity }}+\mathbf{f}^{\text {adsorb }}+\rho \mathbf{g}+\mathbf{f}^{\text {surface }},
$$

where $\mathbf{v}$ and $\mathbf{g}$ denote velocity and gravity, respectively. The pressure force $\mathbf{f}^{\text {pressure }}$ and surface tension $\mathbf{f}^{\text {surface }}$ are computed as in [Müller et al. 2003], and the viscosity force $\mathbf{f}^{\text {viscosity }}$ is computed in a way that takes coagulation into account, as described later on.

Fig. 1 gives an overview of our GPU-based simulation framework, which comprises three main stages: in-vivo simulation, adsorptive $\mathrm{SPH}$, and blood rendering. We design four types of particles: invivo particles, flow particles, adhered particles, and guiding particles; as Fig. 1 shows, each particle type has an optimal position within the framework.

\subsection{In-vivo simulation}

To begin with, we simulate in-vivo blood flow for a diversity of hemorrhages.User-input strokes define the shape of the wound on the skin surface. We can easily change the hemorrhage volume and blood pressure by adjusting the number of in-vivo particles and the momentum of in-vivo acceleration, respectively. Then, we evaluate a simple in-vivo blood flow model within the resulting prismoid, as the in-vivo simulation stage in Fig. 1 shows. In the model, a simple $\mathrm{SPH}$ provides the basis for in-vivo particle evaluation. When the particle collides with an exit polygon, it bleeds as a flow particle.

If we were to omit this in-vivo simulation and simply generate the blood particles randomly over the wound polygons, the densities of the blood particles near the wound polygons would become unstable, and it would be difficult to represent phenomena such as blood splash or streak formation.

\subsection{Coagulation}

After bleeding from the wound, blood immediately starts to contact the air and coagulates through the formation of fibrin. This effect can be represented by changing the viscous force of each flow particle according to the air exposure time. Moving the coagulation timer for a flow particle closer to the surface forward results in faster clot (Fig. 2). In our simulation, we linearly interpolate the coefficient of viscosity between 10 and 100 . Users can change the speed of coagulation via a GUI.
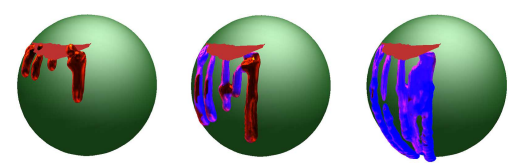

Figure 2: Blood starts to clot after bleeding; to obtain this sequence of images, we changed the normal bloods color to translucent red and the scabs color to opaque blue.

\subsection{Blood rendering}

In line with [Müller et al. 2003], we define the blood surface as an isosurface of a distance field:

$$
\Phi(\mathbf{x})=\sum_{j} \frac{m_{j}}{\rho_{j}} W\left(\mathbf{x}-\mathbf{x}_{j}, h_{j}\right),
$$

where $W$ denotes an isotropic smoothing kernel of the form:

$$
W(\mathbf{r}, h)= \begin{cases}\frac{\sigma}{h^{3}}\left(1-\frac{\|\mathbf{r}\|}{h}\right)^{2} & \text { if }\|\mathbf{r}\| \leq h \\ 0 & \text { otherwise }\end{cases}
$$

where $\sigma$ and $\mathbf{r}$ denote the scaling factor and radial vector, respectively. In practice, we use Marching cubes [Lorensen and Cline 1987] to extract the blood surface.

If we were to consider surface reflection only, we would not be able to represent blood thickness. We render blood as participating media, evaluating light attenuation by accounting for the interaction between light and micro-particles.

Assume that the propagation of light in participating media is denoted by $d L(\mathbf{x}, \vec{\omega})$. The light attenuates due to scattering and absorption within the media. The amount of attenuated light in interval $d s$ is represented as:

$$
d L(\mathbf{x}, \vec{\omega})=-\sigma_{t} L(\mathbf{x}, \vec{\omega}) d s,
$$

where $\sigma_{t}$ denotes the extinction coefficient. This equation, the radiance transport equation (RTE), represents the propagation of light in participating media. Evaluating the RTE increases expensive computational costs for accurate results, so we simplify the equation for real-time rendering of participating media.

We replace $d s$ in Eq. (4) with a longer interval $\Delta s$ and approximate the amount of attenuated light at each vertex $p$ of the blood surface 
meshes in the direction of incident vector $I$ from eye position to $p$ as follows:

$$
\Delta L(p, I)=-\sigma_{t} L(p, I) \Delta s,
$$

where $\Delta s$ is the blood thickness in the direction $I$. We measure blood thickness at each vertex of the blood surface meshes using a distance field.
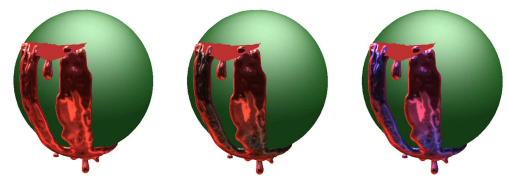

Figure 3: Rendering blood as participating media; when taking participating media into account (middle), blood gets darker than its simple color (left). To illustrate these effects, we have accentuated the thickness in blue (right).

\section{Adsorptive SPH}

This section, which gives detailed descriptions of physisorption as a main source of blood streak formation and its guiding mechanism, contains the primary contributions of the paper. While physisorption is not particular to bleeding, all liquids demonstrate similar features when they flow on an object surface. Therefore, we can extend our directable simulation of streak formation to the behavior of various fluids like the vertical flow of superfluous paint and polluted water on walls.

\subsection{Physisorption}

The phenomena of blood adhering to the surface of the skin and sliding evenly underneath the lower-horizontal surface are types of physisorption caused by van der Waals force. Physisorption is a process in which the electronic structure of an atom or molecule is perturbed upon adsorption [Dash and Stęślicka 1974]. As bleeding develops while the blood is appressed to the skin surface, the forces from the surface have a great influence on the dynamics of the fluid.

\subsubsection{Adsorption force}

We add an adsorption force term, formulated by a quadratic function, to give each flow particle an antigravity nature, as the existing research did [Clavet et al. 2005]:

$$
\mathbf{f}_{i}^{a d s o r b}=-k d_{i}\left(1-\frac{d_{i}}{d^{a d s o r b}}\right) \hat{\mathbf{n}},
$$

where $d_{i}$ denotes the distance between particle $i$ and the skin surface, and $\mathbf{n}$ the surface normal. An attraction force occurs when $d_{i}$ is smaller than $d^{a d s o r b}$. When we add in the adsorption force in Eq. (5), the flow particles manifest their antigravity nature but simply slip through.

To represent adhesion, [Ueda and Fujishiro 2012] formulated an adsorption force based on van der Waals force. A particle near a given object is adsorbed and adhered to the object. However, the volume of the blood represented by particles in SPH is greater than that of adhered blood in reality; hence, the gross volume of blood falls to such a low level that blood is unable to flow smoothly. [Ueda and Fujishiro 2012] thus generated a large amount of blood from the wound and set a low viscosity parameter to enable a smooth flow, but low viscosity can make blood flow hard to ascertain.
To address this problem, we evaluate the adsorption force with Eq. (5) and generate micro-particles around the portions where flow particles collide with the surface. We define these micro-particles as adhered particles, which influence flow particles in terms of pressure and viscosity by summing up the contributions of neighboring adhered particles. As a result, flow particles move smoothly to form traces of blood.

\subsubsection{Saturation of adsorption media}

When a certain number of adsorbents bond with the adsorption media, adsorption force does not act at the saturated position. If we were to ignore this phenomenon, there would be an excess of adhered particles in the same place; this would result in a relatively strong viscosity force acting on the flow particles there. In the worst case, these conditions could terminate the blood flow. To solve the problem, our approach to the saturation of adsorption media assumes that the remaining particles slip through when there are more than a certain number of adhered particles present. [Ueda and Fujishiro 2012] defined the saturation by thresholding the density of indiscriminant particles, whereas our paper refines the definition through the use of smaller-scale adhered particles.

\subsubsection{Distance field for adhered particles}

Defining the adhered blood's surface with Eq. (3) would produce too much adhered blood. If we use a smoothing kernel with a smaller radius, on the other hand, the adhered blood surface may appear jagged because it could potentially remain within a sub-lattice length.

Thus, we use an anisotropic kernel similar to the one proposed in [Yu and Turk 2010] for thin blood surface. The direction of the anisotropic kernel is given by the normal vector on the surface. When defining the adhered blood surface, we re-calculate the radial vector $\mathbf{r}$ as follows:

$$
\mathbf{r}_{\text {new }}=\mathbf{r}+\left(1-\frac{1}{d_{\text {shrink }}}\right)(\mathbf{r} \cdot \mathbf{n}) \mathbf{n},
$$

where $d_{\text {shrink }}$ and $\mathbf{n}$ denote the compression ratio and the directional vector of ellipsoid, respectively. As the radial vector $\mathbf{r}$ in Eq. (6) is updated, $\mathbf{r}$ is stretched along the direction of $\mathbf{n}$. We can easily determine whether the grid point $\mathbf{x}_{i}$ is within the ellipsoidal range or not. Then, we include only grid points whose radial vector $\mathbf{r}_{n e w}$ is smaller than the interaction range $h$ within the ellipsoid. This enables us to represent thin, flat adhered blood, as Fig. 4 illustrates.

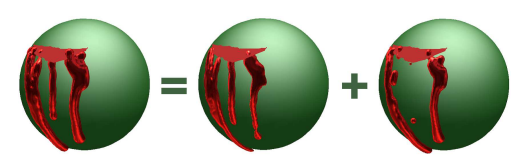

Figure 4: Effects of thin adhered blood; blood (left) is decomposed into adhered blood (middle) and flowing blood (right).

\subsection{Guiding}

When blood streaks on the surface of the skin, the user may want the blood to avoid flowing over important marks and instead arrive at target spots. To facilitate that process, we make use of guiding particles. Our approach allows users to move a cursor along the desired blood flow path and generates particles repeatedly along that track. In more concrete terms, we split the user-input stroke into cylindroid fragments of a certain length. Then, we generate guiding particles while maintaining a constant distance from any 
other guiding particles. The velocities of the generated particles are directed along their cylinder's central axis.

\subsubsection{Guiding force}

Unlike the control particles that [Thürey et al. 2006] proposed, guiding particles remain fixed at their generated positions to help save memory and reduce computational load.

We define the guiding force $\mathbf{f}^{\text {guide }}$ as the viscosity force, which we derive from the velocity $\mathbf{v}_{j}$ of neighboring guiding particles $j \in$ $N_{g}$. If the user wants blood to act against gravity, our approach adds a relatively strong guiding force. If the direction of the guiding force were to shift into the direction of gravity, the particles would move at an exceptionally fast speed. Therefore, we do not simply add the guiding force to the Navier-Stokes equations (2) but rather control the flow particle $i$ by giving the gravity a tilt with guiding force $\mathbf{f}^{\text {guide }}$ defined as:

$$
\mathbf{f}_{i}^{\text {guide }}= \begin{cases}\mu_{g} \sum_{j \in N_{g}} \frac{m_{j}}{\rho_{j}}\left(\mathbf{v}_{j}-\mathbf{v}_{i}\right) \nabla^{2} W(\mathbf{r}, h) \\ \mathbf{g} & \text { if } N_{g} \text { is nonempty } \\ \text { otherwise, }\end{cases}
$$

$$
\mathbf{g}_{i}=\left(1-\alpha_{g}\right) \mathbf{g}+\alpha_{g} \mathbf{f}_{i}^{\text {guide }} .
$$

We set guiding viscosity coefficient $\mu_{g}$ to 10.0 and give the user the ability to control the degree of guiding force $\alpha_{g}$ between 0 and 1 through the GUI (Fig. 5).
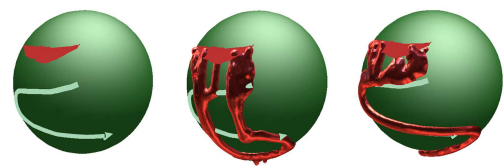

Figure 5: Based on user-input strokes (left), blood is directed depending on the degree of guiding force: respecting physical accuracy (middle) or guiding blood so that it closely matches the strokes (right).

See the accompanying video for four animations corresponding to Figures 2 through 5.

\section{Implementation}

We have relied on the GPU to implement parallel codes for the simulation. In the GPU programming, translating data from the GPU to the CPU would create a bottleneck. By implementing most of the phases on the GPU, as shown in Fig. 1, we have thus minimized the GPU-CPU data transfer volume. For the neighboring particle search process, we have used a hashing method that identifies the first and last indices of neighbor particles.

We establish a pseudo code for "Adsorptive SPH" as follows.

\section{Results and Discussions}

We have performed the bleeding simulation on a standard personal computer with an Intel Xeon E5540 2.53GHz CPU, 12.GB CPUmemory, and a QuadroFX 4800 GPU. We set the simulation time step to $0.001 \mathrm{sec}$. and rendered a frame per three time steps. The HDR (High Dynamic Range) background and the atmospheric blurring effects were from NVIDIA OpenGL SDK 10.0.
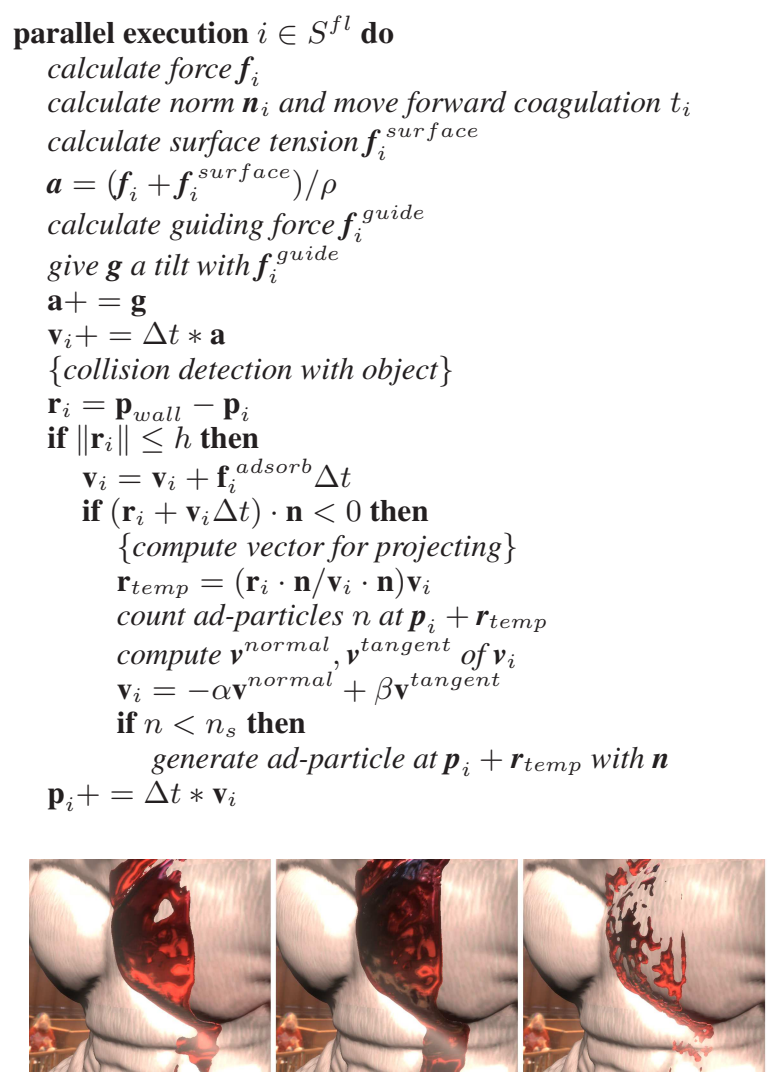

Figure 6: Comparison of smoothing kernels; this representation shows only adhered particles on the armadillo's body. The anisotropic kernel can generate thinner and flatter blood (left) than the isotropic kernel (middle) can. The smaller isotropic kernel just produces jagged results (right).

\subsection{Experimental results}

Fig. 6 shows the effects of anisotropic kernels and participating media on rendering only adhered particles on the armadillo's body. Thin, flat blood is extracted using an anisotropic kernel (left). With an isotropic kernel (middle), blood becomes chunky and bumpy and gets darker than the image on the left because of the thicker blood. For the image on the right, we used another small isotropic kernel to avoid chunky blood. The radius of the kernel is smaller than the side of lattice cell, however, which causes the blood to suffer from aliasing. Note that the image on the left contains a hole on the blood surface due to the accurate distance field, which is impossible to represent with that isotropic kernel which we used for the image in the middle.

Fig. 7 shows the results of a complex topological deformation of a large amount of blood. Big globs of blood falling from above collide with and run down detailed objects like pipes and gears. Note that our method extracts thin, flat blood around adhered particles and renders thick blood extracted from around flow particles in a darker representation.

Fig. 8 compares the present method with previous ones. With the original SPH, blood just slips off the surface. With the method in [Ueda and Fujishiro 2012], an extremely large amount of blood adheres to the bunny surface and thus terminates the streak formation. Only with the adsorption force [Clavet et al. 2005] does the blood manifest its antigravity nature; if we only place microparticles having no influence on flow particles on the surface, there 


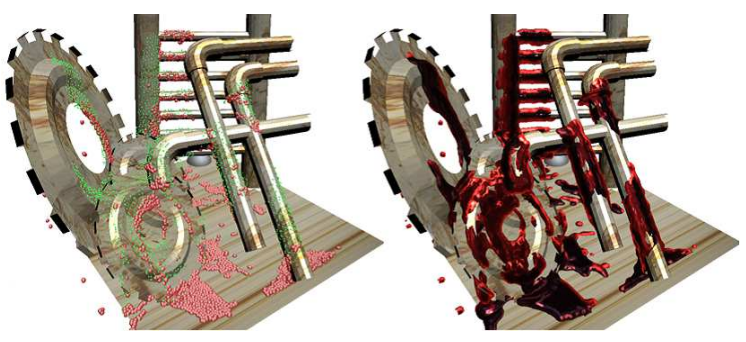

Figure 7: Big globs of blood colliding with and running down objects like pipes and gears; flow particles and adhered particles are colored red and green, respectively (left). We initially generated 7,163 flow particles; after the simulation, there were 4,812 flow particles remaining and 7,266 newly generated adhered particles. These particles are converted into blood meshes using the kernels, which are rendered taking thickness into account (right).

are no streaks, but there is evenly adhered blood. Unlike these results, our method ensures that blood adheres securely onto the surface while generating thin blood streaks. See the accompanying video for more detailed comparisons.

Fig. 9 shows the results of blood guiding in which the user directed bleeding at will by specifying multiple strokes to keep the blood away from the tattoo and flow where he wanted it to go. The accompanying video shows the actual directing process.

\subsection{Simulation time}

Table 1 summarizes the times that it took for the simulation to generate the results in Fig. 9. The grid size for Marching cubes is $128 \times 256 \times 64$. For comparison, we also measured the times for cases where we performed the same simulation entirely on the CPU.

The data transfer from the GPU to the CPU takes substantial time and accounts for most of the total computation time. Our simulation requires two GPU-CPU transfers: the transfer of active voxels during the execution of marching cubes and the transfer of mesh data.

Table 1: The simulation times for generating the results in Fig. 9. Note that ( $\left(a^{\prime}\right)$ shows the result just a few msec. after the time of $(a)$.

\begin{tabular}{|l|r|r|r|r|r|}
\hline & \multicolumn{1}{|c|}{$\left(\mathrm{a}^{\prime}\right)$} & \multicolumn{1}{c|}{ (b) } & \multicolumn{1}{c|}{ (c) } & \multicolumn{1}{c|}{ (d) } & \multicolumn{1}{c|}{$(\mathrm{e})$} \\
\hline \hline Particles & $2.0 \mathrm{k}$ & $3.5 \mathrm{k}$ & $4.3 \mathrm{k}$ & $8.6 \mathrm{k}$ & $16.4 \mathrm{k}$ \\
\hline Meshes & 16,524 & 30,288 & 34,896 & 49,618 & 66,940 \\
\hline \hline Adsorptive SPH [msec] & 1.11 & 2.02 & 2.06 & 2.10 & 2.11 \\
In-vivo simulation [msec] & 0.12 & 0.22 & 0.22 & 0.33 & 0.40 \\
Surface reconstruction [msec] & 0.14 & 0.15 & 0.16 & 0.16 & 0.17 \\
GPU to CPU transfer [msec] & 9.29 & 31.74 & 51.25 & 76.27 & 92.52 \\
\hline Total [fps] & $\mathbf{9 1 . 1}$ & $\mathbf{2 8 . 9}$ & $\mathbf{1 8 . 5}$ & $\mathbf{1 2 . 4}$ & $\mathbf{1 0 . 3}$ \\
\hline \hline Adsorptive SPH (CPU) [msec] & 21 & 31 & 73 & 119 & 130 \\
In-vivo simulation (CPU) [msec] & 32 & 33 & 35 & 37 & 46 \\
Surface reconstruction (CPU) [msec] & 2100 & 4100 & 6100 & 7800 & 9300 \\
\hline Total (CPU) [fps] & $\mathbf{0 . 5}$ & $\mathbf{0 . 2}$ & $\mathbf{0 . 2}$ & $\mathbf{0 . 1}$ & $\mathbf{0 . 1}$ \\
\hline
\end{tabular}

\subsection{Limitations}

Under the current implementation of the codes, it is impossible for the user to control the bleeding along the timeline of a scenario. However, it may be possible to enable this functionality by extending the GUI so that the guiding particles can effectively coordinate their respective velocities.
As it currently stands, we cannot distinguish between artery blood and venous blood in terms of physiological properties and appearance. However, one could easily argue that adjusting the viscosity and attenuation rate $F$ parameters as functions of the concentration of red blood cells would help discriminate between the two types.

\section{Conclusion}

We have proposed a directable simulation of bleeding in real time on the GPU and proved that a SPH-based fluid simulation can represent phenomena peculiar to bleeding: adherence onto the skin surface and streak formation on the surface. To enable directable bleeding, we have defined guiding particles that allow us to control the trajectories of blood flow freely. The present SPH may find applications in similar phenomena like paint art and extend easily to related methods such as PCISPH to enrich the applicable content in virtual worlds.

\section{Acknowledgements}

We thank the anonymous reviewers for their helpful feedbacks. This work has been partially supported by JSPS KAKENHI Grant Number 26240015.

\section{References}

Adams, B., Pauly, M., Keiser, R., and Guibas, L. J.: "Adaptively sampled particle fluids," ACM Transactions on Graphics, Vol. 26, No. 3, Article 48, 2007.

Amada, T., Imura, M., Yasumuro, Y., Manabe, Y., and Chihara, K.: "Particle-based fluid simulation on GPU," in Proceedings of ACM Workshop on General-Purpose Computing on Graphics Processors, 2004.

Clavet, S., Beaudoin, P., and Poulin, P.: "Particle-based viscoelastic fluid simulation," in Proceedings of the 2005 ACM SIGGRAPH/Eurographics Symposium on Computer Animation, pp. 219-228, 2005.

Daenzer, S., Montgomery, K., Dillmann, R., and Unterhinninghofen, R.: "Real-time smoke and bleeding simulation in virtual surgery," Studies in Health Technology and Informatics, Vol. 125, pp. 94-99, 2006.

Dash, J. G. and Stęślicka, M.: "Statistical thermodynamics of physisorption; Kronig-Penney model for surface states," Progress in Surface Science, Vol. 5, Part 2, 1974.

Desbrun, M. and Gascuel, M. P.: "Smoothed Particles: A new paradigm for animating highly deformable bodies," in Proceedings of Eurographics Workshop on Computer Animation and Simulation, pp. 61-76, 1996.

Frisvad, J. R., Christensen, N. J., and Jensen, H. W.: "Computing the scattering properties of participating media using LorenzMie theory," ACM Transactions on Graphics, Vol. 26, No. 3, Article 60, 2007.

Gingold, R. and Monaghan, J.: "Smoothed Particle Hydrodynamics - Theory and application to nonspherical stars," Monthly Notices of the Royal Astronomical Society, Vol. 181, pp. 375-389, 1977.

Goswami, P., Schlegel, P., Solenthaler, B., and Pajarola, R.: "Interactive SPH simulation and rendering on the GPU," in Proceedings of the 2010 ACM SIGGRAPH/Eurographics Symposium on Computer Animation, pp. 55-64, 2010. 


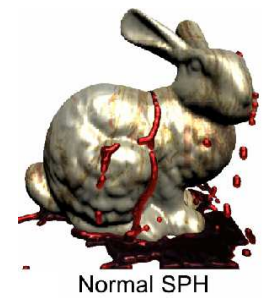

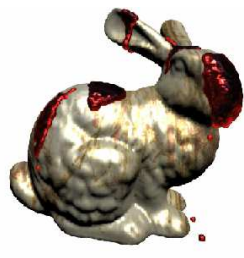

Ueda and Fujishiro [1]

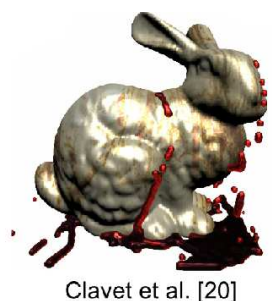

Clavet et al. [20]

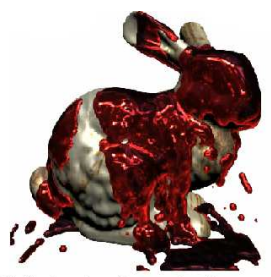

[20] \& Just adhered micro-particles

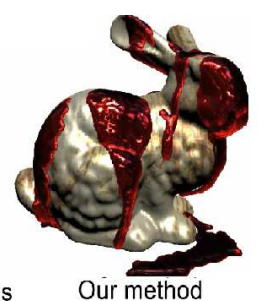

Our method

Figure 8: A comparison with previous works; big globs of blood run down the bunny surface. With the original SPH, blood slips off the bunny. Under the method in [Ueda and Fujishiro 2012], a large amount of blood terminates streak formation. Only with the adsorption force [Clavet et al. 2005] does the blood manifests its antigravity nature, but it just slips through. If we only place micro-particles having no influence on flow particles on the surface, there are no streaks, but there is evenly adhered blood. Unlike these methods, our method generates thin blood streaks on the surface.

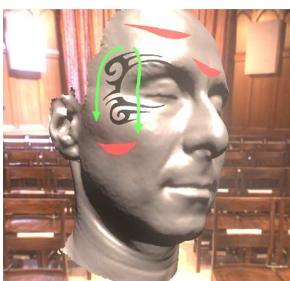

(a)

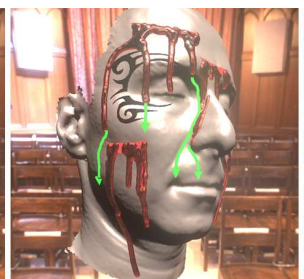

(b)

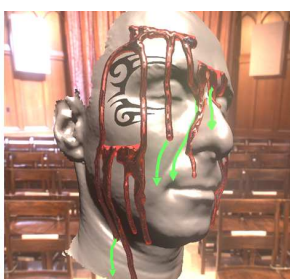

(c)

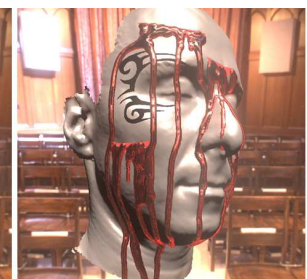

(d)

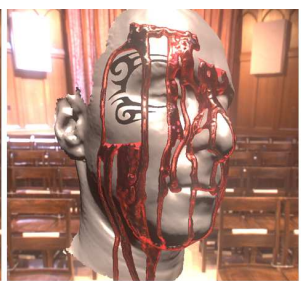

(e)

Figure 9: The result of directing bleeding interactively; the user has the ability to direct bleeding and keep the blood away from the tattoo or prevent overlapping among the bleeding traces by guiding blood along the user-input strokes. See the accompanying video for more details. The video illustrates the complicated merge and split patterns and dynamics of blood streaks reflecting the face's and wounds' geometries. Table 1 shows the simulation times.

Harada, T., Koshizuka, S., and Kawaguchi, Y.: "Smoothed particle hydrodynamics on GPUs," in Proceedings of Computer Graphics International 2007, pp. 63-70, 2007.

Harada, T., Koshizuka, S., and Kawaguchi, Y.: "Sliced data structure for particle-based simulations on GPUs," in Proceedings of 5th International Conference on Computer Graphics and Interactive Techniques in Australia and Southeast Asia, pp. 55-62, 2007.

Ihmsen, M., Akinci, N., Becker, M., and Teschner, M.: "A parallel SPH implementation on multi-core CPUs," Computer Graphics Forum, Vol. 30, No. 1, pp. 99-112, 2011.

Kim, Y., Machiraju, R., and Thompson, D.: "Path-based control of smoke simulations," in Proceedings of the 2006 ACM SIGGRAPH/Eurographics Symposium on Computer Animation, pp. 33-42, 2006.

Lorensen, W. E. and Cline, H. E.: "Marching Cubes: A high resolution 3D surface construction algorithm," in Proceedings of ACM SIGGRAPH 1987, pp. 163-169, 1987.

Lucy, L.: "A numerical approach to the testing of the fission hypothesis," Astronomical Journal, Vol. 82, pp. 1013-1024, 1977.

Miller, G. and Pearce, A.: "Globular Dynamics: A connected particle system for animating viscous fluids," Computers and Graphics, Vol. 13, No. 3, pp. 305-309, 1989.

Müller, M., Charypar, D., and Gross, M.: "Particle-based fluid simulation for interactive applications," in Proceedings of the 2003 ACM SIGGRAPH/Eurographics Symposium on Computer Animation, pp. 154-159, 2003.

Müller, M., Schirm, S., and Teschner, M.: "Interactive blood simulation for virtual surgery based on smoothed particle hydro- dynamics," ACM Technology and Health Care, Vol. 12, No. 1, pp. 25-31, 2004.

Morris, J. P.: "Simulating surface tension with smoothed particle hydrodynamics," International Journal for Numerical Methods in Fluids, Vol. 33, No. 3, pp. 333-353, 2000.

Reeves, W. T.: "Particle System - A technique for modeling a class of fuzzy objects," ACM Transactions on Graphics, Vol. 2, No. 2, pp. 359-376, 1983.

Solenthaler, B. and Pajarola, R.: "Predictive-corrective incompressible SPH," ACM Transactions on Graphics, Vol. 28, No. 3, Article 40, 2009.

Schechter, H. and Bridson, R.: "Ghost SPH for animating water," ACM Transactions on Graphics, Vol. 31, No. 4, Article 61, 2012.

Solenthaler, B. and Gross, M.: "Two-scale particle simulation," ACM Transactions on Graphics, Vol. 30, No. 4, Article 81, 2011.

Thürey, N., Keiser, R., Pauly, M., and Rüde, U.: "Detailpreserving fluid control," in Proceedings of the 2006 ACM SIGGRAPH/Eurographics Symposium on Computer Animation, pp. 7-12, 2006.

Ueda, K. and Fujishiro, I.: "Visual simulation of bleeding on skin surfaces," in Proceedings of Computer Graphics International 2012, 2012.

Wang, H., Mucha, P. J., and Turk, G.: "Water drops on surfaces," ACM Transactions on Graphics, Vol. 24, No. 3 , pp. 921-929, 2005.

Wang, H., Miller, G., and Turk, G.: "Solving general shallow wave equations on surfaces," Proceedings of the 2007 ACM SIGGRAPH/Eurographics Symposium on Computer Animation, pp. 229-238, 2007. 
Xue-mei, L., Ai-min, H., Qin-ping, Z., and Chun-yan, C.: "Bleeding simulation based particle system for surgical simulator," in Proceedings of 2009 Pacific-Asia Conference on Knoeledge Engineering and Software Engineering, pp. 151-154, 2009.

Yim, D., Baranoski, G. V. G., Kimmel, B. W., Chen, T. F., and Miranda, E.: "A cell-based light interaction model for human blood," Computer Graphics Forum, Vol. 31, No. 2, pp. 845-854, 2012.

Yu, J. and Turk, G.: "Reconstructing surfaces of particle-based fluids using anisotropic kernels," in Proceedings of the 2010 ACM SIGGRAPH/Eurographics Symposium on Computer Animation, pp. 217-225, 2010.

Zhang, Y., Wang, H., Wang, S., Tong, Y., and Zhou, K.: "A deformable surface model for real-time water drop animation," IEEE Transactions on Visualization and Computer graphics, Vol. 99, 2011.

Zhang, Y., Solenthaler, B., and Pajarola, R.: "Adaptive sampling and rendering of fluids on the GPU," in Proceedings of Eurographics/IEEE VGTC Symposium on Point-Based Graphics, pp. 137-146, 2008.

Zhou, K., Hou, Q., Wang, R., and Guo, B.: "Real-time KD-tree construction on graphics hardware," ACM Transactions on Graphics, Vol. 27, No. 5, pp. 1-11, 2008. 